\title{
Tratamiento restaurador atraumático para niños hospitalizados
}

\section{Resumen}

Objetivos: No se sabe si el tratamiento restaurador atraumático (TRA) es posible en niños de bajo nivel socioeconómico internados en hospitales públicos. Este estudio retrospectivo abordó la aceptación del TRA por los pacientes y por el equipo odontológico en la enfermería pediátrica del Hospital das Clínicas da Universidade Federal de Goiás, en Goiânia, Brasil. Material y métodos: Investigamos la técnica del TRA bajo la óptica del niño hospitalizado que recibió el tratamiento durante el internamiento, relatos de los acompañantes y de los operadores que ejecutaron la técnica. Resultados: El TRA fue realizado en 35 dientes de 18 niños. Dieciocho casos se relacionaron a "comodidad", 12 a "incomodidad mínima" y 5 el "dolor moderado". El comportamiento de los niños fue "definiti-

\author{
Rezende $L^{1}$ \\ Daher $C^{2}$ \\ Tolêdo $R^{3}$ \\ Araujo de Oliveira $A^{4}$
}

vamente positivo" ( $\mathrm{n}=10)$ o "positivo" $(\mathrm{n}=25)$. El sentimiento de los niños no se alteró tras el procedimiento $(\mathrm{P}=0,358)$ y su percepción sobre TRA fue más favorable cuando eran mayores, tenían experiencia anterior con el tratamiento odontológico, no relataron dolor durante el procedimiento y el TRA fue realizado más rápido $(\mathrm{P}<0,05)$. Los acompañantes $(80,0 \%)$ tuvieron opinión positiva acerca del TRA. Los operadores no relataron ninguna dificultad en realizar 48,6\% de los casos de TRA. Conclusión: Como el TRA tuvo buena aceptación por los pacientes pediátricos, acompañantes y operadores, puede ser una acción de programas con miras a la población carente hospitalizada.

Palabras clave: unidad hospitalaria de odontología, odontopediatría, asistencia odontológica para enfermos crónicos.

\section{Artigo Original}

\section{Tratamento restaurador atraumático para crianças hospitalizadas}

\section{Resumo}

Objetivos: Não se sabe se o tratamento restaurador atraumático (ART) é viável em crianças de baixo nível socioeconômico internadas em hospitais públicos. Este estudo retrospectivo abordou a aceitação do ART por pacientes e pela equipe odontológica na efermaria pediá-

\footnotetext{
${ }^{1}$ Doctora en Ciencias Odontológicas, área de concentración Odontopediatría; Profesora Asociada de la Faculdade de Odontologia da Universidade Federal de Goiás (UFG), Goias, Brasil

${ }^{2}$ Especialista en Odontopediatria, cirurgiã-dentista de la Secretaría Municipal de Saude, Anápolis-Goias, Brasil

${ }^{3}$ Doctora en Odontologia, Profesora Adjunta de la Faculdade de Odontologia da Universidade Federal de Juiz de Fora,Minas Gerais, Brasil

${ }^{4}$ Master en Odontologia, cirurgiã-dentista del Hospital de Clínicas da UFG, Goias, Brasil
} 
trica do Hospital das Clínicas da Universidade Federal de Goiás, em Goiânia, Brasil. Material e métodos: Investigamos a técnica do ART sob a óptica da criança hospitalizada que recebeu o tratamento durante a internação, relatos dos acompanhantes e dos operadores que executaram a técnica. Resultados: O ART foi realizado em 35 dentes de 18 crianças. Dezoito casos relacionaram-se a "conforto", 12 a "desconforto mínimo" e 5 a "dor moderada". O comportamento das crianças foi "definitivamente positivo" $(\mathrm{n}=10)$ ou "positivo" $(\mathrm{n}=25)$. O sentimento das crianças não se alterou depois do procedimento $(\mathrm{P}=0,358)$ e sua percepção sobre ART foi mais favorável quando eram mais velhas, tinham expe- riência anterior com tratamento odontológico, não relataram dor durante o procedimento e o ART foi realizado mais rapidamente $(P<0,05)$. Acompanhantes $(80,0 \%)$ tiveram opinião positiva a respeito do ART. Operadores não relataram nenhuma dificuldade em realizar 48,6\% dos casos de ART. Conclusão: Como o ART teve boa aceitação pelos pacientes pediátricos, acompanhantes e operadores, pode ser uma ação de programas voltados à população carente hospitalizada.

Palavras chave: unidade hospitalar de odontologia, odontopediatria, assistência odontológica para doentes crônicos.

Original article

\section{Atraumatic restorative treatment for hospitalized children}

\begin{abstract}
Objectives: The atraumatic restorative treatment (ART) has been recommended to restore teeth of people who would not normally have access to dental care, but it is not known if general hospitals would be a favorable setting to carry on ART. This retrospective study assessed the acceptance of the ART by patients and dental personnel in the Pediatric Ward of a University Hospital, Brazil. Material and methods: We analyzed 105 dental charts of children admitted to the hospital regarding child's pain/discomfort (observational scale), behavior (Frankl scale), and perception of ART (faces scale), beside accompanying adults and operators reports on ART. Results: ART was performed in 35 teeth of 18 children. Observers classified 18 cases as 'comfort', 12 'minimal discomfort', and 5 'moderate pain'. Children's behavior was
\end{abstract}

'definitely positive' ( $n=10)$ or 'positive' ( $n=25)$. FRS scores did not change after the procedure $(1.9 \pm 1.5, \mathrm{P}=0.358)$. Children's perception on ART was more favorable if they were older, had previous experience of dental treatment, did not report pain during the procedure, and had the procedure performed faster when compared with other ARTs from the same group $(\mathrm{P}<0.05)$. Accompanying persons $(80.0 \%)$ had positive opinions of ART. Operators did not find any difficulty in performing $48.6 \%$ of ARTs. Conclusion: ART had a good acceptance by pediatric patients, accompanying persons and dental care providers. Health programs directed to low income population in hospital settings can include ART in interdisciplinary educational programs focusing on oral health.

Key words: Dental service hospital, pediatric dentistry, dental care for chronically ill 


\section{Introducción}

La relación entre salud oral, salud general y calidad de vida ${ }^{1}$ debe ser reconocida por los educadores del área de la salud, profesionales de salud, gestores y comunidad. Según la Organización Mundial de la Salud (OMS), para mejorar de la salud oral es necesario prevenir las enfermedades orales e integrar la promoción de la salud oral con la prevención de enfermedades crónicas y promoción de la salud en general, pues los riesgos para la salud están asociados ${ }^{1}$.

El cuidado con la salud oral, en el Brasil, ha sido promovido por el Sistema Único de Saúde (SUS) e incluye la atención primaria (Programa Estratégia Saúde da Família) y cuidados terciarios (Centros de Especialidades Odontológicas) ${ }^{2,3}$. El SUS es reconocido como política social biensucedida ${ }^{4}$, pero el acceso a los servicios de salud por la población brasileña aún es un desafío. Aunque el predominio y la gravedad de las caries dentales muestren tendencia de reducción en el periodo de 1980 a 2003, el índice de dientes cariados, obturados y perdidos (COP-D) está dentro de la franja moderada: sólo $28 \%$ de los niños con COP-D $\geq 6$ recibieron atención odontológica ${ }^{5}$.

Una de las estrategias de la OMS para el Programa Global de Salud Oral está dirigida para las Escuelas Promotoras de Salud ${ }^{1}$. De acuerdo con el concepto relativamente nuevo de promoción de la salud para niños y adolescentes en hospitales $^{6}$, otro escenario posible para los cuidados de salud oral es el ambiente hospitalario. Niños internados pueden recibir orientaciones sobre salud oral mientras están internadas en enfermerías pediátricas ${ }^{7,8}$. Además, esos niños pueden beneficiarse con el Tratamiento Restaurador Atraumático (TRA) realizado en el propio lecho del paciente encamado. El TRA es un procedi- miento mínimamente invasivo, no dependiente de equipamientos odontológicos o electricidad y que puede ser usado en diferentes ambientes para mejorar la salud oral de niños de bajo nivel socio-económico 9 . El TRA tiene una tasa de sobrevida igual o mayor que las restauraciones de amalgama hasta por 6,3 años, especialmente considerando restauraciones de una superficie $^{10,11}$, que dependen: del individuo ${ }^{9}$, tipo del diente (permanente/primario) ${ }^{11}$, localización del diente ${ }^{11}$, tamaño de la cavidad ${ }^{9,12}$, viscosidad del cemento de ionómero de vidrio ${ }^{13}$ y experiencia del operador ${ }^{10}$.

La técnica TRA fue recomendada por la OMS para restaurar los dientes de personas que no tienen acceso al tratamiento odontológico convencional ${ }^{14}$. Al parecer no existen estudios acerca del TRA en niños hospitalizados. El objetivo de este estudio fue evaluar la aceptación del tratamiento TRA en los niños con enfermedad sistémica internados en un hospital universitario, revisando la aceptación del TRA por los pacientes, sus acompañantes adultos y los operadores del TRA (estudiantes de Odontología).

\section{Material y Métodos}

Población y muestra

Este estudio retrospectivo incluyó 105 fichas odontológicas de niños que frecuentaron la enfermería pediátrica del Hospital de Clínicas da Universidade Federal de Goiás (UFG), en el estado de Goiás, durante 10 meses. La UFG es uno de los principales hospitales universitarios del Centro-Oeste/Oeste del Brasil y tiene convenio con el SUS para atención terciaria de media y alta complejidad. Tiene una capacidad total de 310 lechos, siendo que la enfermería pediátrica posee 25 lechos y atiende pacientes de bajo nivel socio-económico del estado de Goiás y estados 
vecinos. Los niños son acompañados por un adulto durante la internación; los acompañantes permanecen junto al lecho durante toda la estada en el hospital, como previsto por la ley, participando activamente de la recuperación del niño. El ambiente hospitalario en el Brasil es generalmente desconocido por los estudiantes de Odontología considerando acciones de promoción y educación en salud oral; ya que las actividades de los alumnos en hospitales son generalmente restrictas a las cirugías.

Este grupo de niños participó de un programa piloto en salud oral, que comprendió medidas educativas, preventivas y restauradoras, coordinado por la Facultad de Odontología de la UFG en colaboración con el sector de odontología del hospital. Un grupo de ocho estudiantes del quinto año y dos alumnos de Posgrado atendieron a los niños hospitalizados y a sus acompañantes adultos, cuatro horas por semana, durante 10 meses. Los estudiantes, entrenados para la realización del TRA (protocolo de la $\left.\mathrm{OMS}^{15}\right)$, realizaron TRA mientras el niño estaba acostado en una cama de hospital, bajo luz natural, utilizando instrumentos manuales y restaurando los dientes con cemento de ionómero de vidrio de media viscosidad. El procedimiento odontológico era realizado a 4 manos. Niños que necesitaron de extracción dentaria fueron encaminados a un consultorio convencional en el hospital. Los padres de los niños que recibieron alta del hospital fueron orientados a buscar los servicios odontológicos en sus comunidades.

Este estudio fue aprobado por el Comité de Ética en Investigación del Hospital de Clínicas da UFG, siguiendo las recomendaciones de la Resolución 196/96 del Conselho Nacional de Saúde-Brasil.

\section{Colección de datos}

Un autor (CDC) recolectó informaciones sobre la salud sistémica y odontológica de los niños a partir de las fichas, tales como: edad, sexo, historia de tratamiento odontológico anterior y el índice coe-d/COP-D. Recolectó también informaciones detalladas sobre el TRA realizado en los niños:

- Número y tipo de dientes, extensión de la cavidad, tiempo necesario para la excavación de la dentina y para la conclusión del procedimiento.

- Relatos de los niños sobre dolor y sentimiento relacionados a la realización del TRA. El dolor, durante la realización del TRA, era registrada como \"sí \" o \"no", y el sentimiento del niño con base en una escala de 6 caras cuyos valores variaron de 0 ("muy bueno") a 5 ("muy malo").

- Medidas observacionales de evaluación del dolor y comportamiento del niño. Para cada procedimiento TRA, se evaluó el dolor del niño según la escala "Sounds, Eye and Motor" (SEM) ${ }^{16}$ (Cuadro 1), y su comportamiento conforme la escala de Frank ${ }^{17}: 1$ - niño es completamente cooperativo y hasta le gusta la experiencia; 2 - niño es cooperativo, pero un poco reluctante/tímido, 3 - niño es terco, muy reluctante en oír/responder las preguntas, sin embargo algún progreso es posible; 4 - niño completamente no colabora, llora bastante.

- Relatos de los estudiantes de odontología y de los acompañantes del niño sobre la experiencia con el TRA, los cuales fueron categorizados como \"positiva \" o \"negativa \". 
Cuadro 1. Criterios para la puntuación del dolor del niño según la escala SEM ("sound, eyes, motor").

\begin{tabular}{|c|c|c|c|c|}
\hline \multirow{2}{*}{$\begin{array}{l}\text { Observaciones de } \\
\text { posibles indica- } \\
\text { ciones de dolor }\end{array}$} & \multicolumn{4}{|c|}{ Valores para nivel de comodidad o dolor * } \\
\hline & 0 - Confortable & $\begin{array}{l}1 \text {-incomodidad } \\
\text { mínima }\end{array}$ & $\begin{array}{c}2 \text { - Moderadamente } \\
\text { doloroso }\end{array}$ & 3 - Doloroso \\
\hline Sonido & $\begin{array}{l}\text { Ningún sonido } \\
\text { indicando dolor }\end{array}$ & $\begin{array}{l}\text { Sonido no especí- } \\
\text { fico; posible indi- } \\
\text { cación de dolor }\end{array}$ & $\begin{array}{l}\text { Reclamación verbal } \\
\text { específica (cómo } \\
\text { "Ay"), levanta la voz }\end{array}$ & $\begin{array}{l}\text { Queja verbal } \\
\text { indica dolor } \\
\text { intenso (cómo } \\
\text { gritando, sollo- } \\
\text { zando) }\end{array}$ \\
\hline Ojo & $\begin{array}{l}\text { Sin señales ocu- } \\
\text { lares de dolor }\end{array}$ & $\begin{array}{l}\text { Ojos abiertos: } \\
\text { demostración de } \\
\text { preocupación, sin } \\
\text { lágrimas }\end{array}$ & $\begin{array}{l}\text { Ojos llorosos, inse- } \\
\text { guros }\end{array}$ & $\begin{array}{l}\text { Lloro, lágrimas } \\
\text { corriendo por el } \\
\text { rostro }\end{array}$ \\
\hline Motor & $\begin{array}{l}\text { Manos relajadas: } \\
\text { ninguna tensión } \\
\text { corporal apa- } \\
\text { rente }\end{array}$ & $\begin{array}{l}\text { Manos mostrando } \\
\text { alguna angustia o } \\
\text { tensión, se agarra } \\
\text { en la silla debido } \\
\text { a incomodidad, } \\
\text { tensión muscular }\end{array}$ & $\begin{array}{l}\text { Movimiento aleatorio } \\
\text { de brazos o cuerpo } \\
\text { sin intención agresiva } \\
\text { del contacto físico, } \\
\text { hace muecas, se re- } \\
\text { tuerse }\end{array}$ & $\begin{array}{l}\text { Movimiento de } \\
\text { las manos para } \\
\text { hacer contacto } \\
\text { físico agresivo } \\
\text { (como puñadas, } \\
\text { estirar la cabe- } \\
\text { za) }\end{array}$ \\
\hline
\end{tabular}

* Cada niño recibe una puntuación para sonido, ojo y parámetros motores; la suma de esos valores es la puntuación SEM total (mínimo 0, máximo 9)

\section{Análisis estadístico}

Se utilizó estadística descriptiva para síntesis de los datos. Las pruebas Kruskal-Wallis y correlación de Spearman asociaron las variables dependientes (SEM, Frankl y valores final en la escala de caras) con las variables independientes del niño (sexo, edad y tratamiento dentario anterior) y el procedimiento de TRA (primer valor en la escala de caras, periodo de tiempo necesario para excavación de la dentina y para la conclusión del procedimiento, dolor relatado por el niño). La prueba de Wilcoxon comparó la puntuación inicial y final en la escala de caras.
Para todas las escalas utilizadas, la mayor puntuación representó más opiniones o comportamientos negativos. Se utilizó el programa SPSS Statistics 17.0 para analizar los datos, considerando alfa de 0,05.

\section{Resultados}

De un total de 105 fichas odontológicos, fueron excluidos 15 de niños edéntulos y 16 de niños que no recibieron examen físico intraoral (por estar durmiendo o fuera del lecho). De los 74 prontuarios incluidos, $34(45,9 \%)$ representaban 
niños con experiencia de caries dental cuyo índice coe-d / COP-D varió de 1 a 20 (media de 4,7): 27 dientes con caries, $13(17,6 \%)$ dientes restaurados y $3(4,1 \%)$ dientes extraídos o ausentes. Entre los 27 niños que presentaran caries dental en el momento del examen, 18 recibieron TRA en 35 dientes, o sea, $66,6 \%$ de los niños tuvieron sus necesidades restauradoras atendida por el TRA. Esos comprendieron 7 niños y 11 niñas de 2,8 a 13,3 años (media \pm desvío padrón $/ \mathrm{DP}=9,0 \pm 3,1$ ), y
4 de ellos ya habían ido al dentista en el pasado. El TRA fue realizado en 25 dientes primarios y 10 dientes permanentes; envolvió cavidades de una superficie oclusal de premolares y molares $(n=19)$, de superficies oclusales y proximales de dientes posteriores $(n=15)$, o de una superficie proximal de dientes anteriores $(n=1)$. Considerando cada diente individualmente, la excavación de la dentina cariada fue realizada en 4,1 $\pm 3,2$ minutos (media \pm DP), y todo el procedimiento de TRA

Tabla 1. Asociación entre variables independientes, dolor y comportamiento durante realización del TRA y sentimiento del niño después de la realización del TRA

\begin{tabular}{|c|c|c|c|}
\hline Variables independientes & $\begin{array}{c}\text { Dolor } \\
\left(\text { Valores } \mathrm{SEM}^{*}\right)\end{array}$ & $\begin{array}{l}\text { Comportamiento } \\
\text { (Valores Franklt) }\end{array}$ & $\begin{array}{l}\text { Sentimiento des- } \\
\text { pués del el TRA } \\
\text { (Valores caras } \ddagger \text { ) }\end{array}$ \\
\hline \multicolumn{4}{|l|}{ Sexo } \\
\hline Femenino & $1,8 \pm 0,9$ & $1,7 \pm 0,5$ & $2,0 \pm 1,6$ \\
\hline Masculino & $1,5 \pm 0,5$ & $1,8 \pm 0,4$ & $1,7 \pm 1,5$ \\
\hline Valor-P & 0,047 & 0,527 & 0,405 \\
\hline \multicolumn{4}{|l|}{$\begin{array}{l}\text { Experiencia anterior con } \\
\text { tratamiento dental }\end{array}$} \\
\hline No & $1,9 \pm 0,8$ & $1,8 \pm 0,4$ & $2,5 \pm 1,2$ \\
\hline $\mathrm{Si}$ & $1,5 \pm 0,7$ & $1,7 \pm 0,5$ & $1,4 \pm 1,6$ \\
\hline Valor-P & 0,397 & 0,586 & $<0,001$ \\
\hline \multicolumn{4}{|c|}{$\begin{array}{c}\text { Dolor durante el procedimiento } \\
\text { TRA (auto-relato) }\end{array}$} \\
\hline No & $1,6 \pm 0,7$ & $1,7 \pm 0,5$ & $1,7 \pm 1,4$ \\
\hline $\mathrm{Si}$ & $2,0 \pm 1,2$ & $1,5 \pm 0,6$ & $3,0 \pm 2,3$ \\
\hline Valor-P & 0,066 & 0,320 & 0,001 \\
\hline
\end{tabular}

* "Sound, eye, motor", mínimo 0, máximo 9

+ Mínimo 1, máximo 4

¥Escala de caras, mínimo 0, máximo 5

$\S P<0,01$ 
Tabla 2. Correlaciones entre variables cuantitativas

\begin{tabular}{|c|c|c|c|c|c|}
\hline Variables & $(1)$ & $(2)$ & $(3)$ & $(4)$ & (5) \\
\hline (1) Edad (meses) & - & - & & \\
\hline $\begin{array}{c}\text { (2) Tiempo necesario para excavación de } \\
\text { dentina }\end{array}$ & 0,25 & - & & \\
\hline (3) Tiempo necesario para conclusión del \\
TRA & $0,47 \ddagger$ & $0,74 \neq$ & - & - & - \\
\hline (4) Escala SEM & $-0,40 \S$ & $-0,33$ & $-0,21$ & $0,59 \neq$ & $0,44 \neq$ \\
\hline (5) Escala Frankl & $-0,23$ & $-0,12$ & 0,07 & 0,21 & $0,40 \neq$ \\
\hline (6) Sentimiento antes del TRAt & $-0,17$ & $-0,08$ & $-0,12$ & 0,07 & \\
\hline (7) Sentimiento después del TRA & $-0,38 \S$ & $-0,05$ & $-0,36 \S$ & & \\
\hline
\end{tabular}

* "Sound, eye, motor", mínimo 3, máximo 12

+ Escala de caras, mínimo 0, máximo 5

$\neq P<0,05$

$\S P<0,01$

fue concluido en 7,5 $\pm 4,7$ minutos (media $\pm \mathrm{DP}$ ). Hubo relato de dolor en 4 de los 35 casos.

En de acuerdo con la escala SEM, durante la realización del TRA, 18 casos fueron clasificados como "confortable", 12 "mínimo de incomodad" y 5 "dolor moderado", esta última observada solamente en el grupo del género femenino. Los valores SEM variaron significativamente con el sexo y la edad (Tablas 1 y 2). El comportamiento de los niños (escala de Frankl), durante la realización del TRA, fue clasificado como "definitivamente positivo" ( $\mathrm{n}=10)$ y "positivo" $(\mathrm{n}=25)$, pero ninguna de las variables independientes analizadas se asoció significativamente con el comportamiento del niño (Tablas 1 y 2).

Por los valores en la escala de caras, los niños estaban sintiéndose razonablemente bien antes del procedimiento $(2,2 \pm 1,8$, media $\pm \mathrm{DP})$. Sus sentimientos no se alteraron significativamente después del procedimiento de TRA (valores 1,9 $\pm 1,5, \mathrm{P}=0,358$, Wilcoxon Signed Rank Test). Los niños se sintieron mejor después del TRA (valores bajos en la escala de caras) cuando eran más viejas, tenían experiencia anterior de tratamiento dentario, no relataron dolor durante el procedimiento y tuvieron el procedimiento realizado más rápidamente (Tablas 1 y 2 ).

En 80,0\% de los casos, los acompañantes estaban satisfechos con el TRA y relataron aspectos positivos de la técnica: buen comportamiento de los niños pues el TRA no incluye agujas o anestesia local $(n=7)$; no haber gastos con el tratamiento, una vez que fue financiado por el Sistema Único de Saúde $(n=4)$. Los otros $20,0 \%$ no se sintieron aptos a opinar. 
Los estudiantes de Odontología no encontraron ningún problema en la ejecución de 48,6\% $(\mathrm{n}=17)$ de los casos de TRA, pero pequeñas dificultades fueron relatadas por los otros $51,4 \%$ $(n=18)$ : en el acceso las lesiones de caries $(n=8)$, en la iluminación externa $(n=6)$, en el comportamiento no cooperativo del niño $(n=2)$ y en la posición del operador $(n=2)$.

\section{Discusión}

"Primum non nocere $\backslash$ ". Nuestros resultados indican que un programa de salud oral en el ambiente hospitalario público, que incluya el enfoque TRA, está de acuerdo con la idea de proporcionar \"mejores cuidados con empatía y humanización \" para niños ${ }^{18}$.

El grupo que se sintió mejor después del TRA incluyó niños con características más favorables en cuanto a la edad, historia de tratamiento odontológico, dolor y comportamiento durante el procedimiento, y tiempo de ejecución del TRA. Esos resultados eran esperados. Una revisión sistemática ${ }^{19}$ mostró que el TRA minimiza el dolor relatado por los pacientes y tiene características favorables para los pacientes que sufren de miedo o ansiedad odontológicos. Además de eso, era esperado que tiempos más largos para la ejecución del TRA estuvieran relacionados con cavidades mayores, pues fue relatado en otro estudio ${ }^{20}$ que el dolor, durante TRA, es más observado en cavidades extensas y con pared cerca de la pulpa. También, los niños que tuvieron tratamiento odontológico previo podrían comprender concretamente las ventajas de no tener la punción de la aguja, el ruido de pieza de mano y los otros estímulos negativos del tratamiento convencional. Infelizmente, como este fue un estudio retrospectivo, no se evaluó el tamaño de la cavidad antes de la realización de la restauración.
La incomodad o dolor observados durante el TRA (escala SEM) fueron generalmente leves y significativamente influenciados por la edad del niño (correlación inversa). Los valores SEM tendieron a asociarse al auto-relato de dolor por los niños, pero esa relación no fue significativa, tal vez debido a un error tipo II (el pequeño número de la muestra puede fallar para negar la hipótesis nula cuando los grupos de comparación son diferentes). Además, se verificó correlación moderada significativa de los valores SEM con el comportamiento de los niños en la escala de Frankl, la cual también fue correlacionada con el sentimiento del niño antes y después del TRA. Este resultado sugiere que parte del comportamiento y sentimientos de los niños, en esos casos, podría estar relacionado la diferentes razones, tales como: características de personalidad/ carácter del niño o experiencias traumáticas con hospitalización ${ }^{21}$. Los valores de Frankl, sin embargo, fueron bajos, indicando cooperación de los niños durante la realización del TRA.

Este estudio demostró que los estudiantes, incluso entrenados, tuvieron algunos problemas relacionados al método TRA y al comportamiento de los niños. Tal vez si fueran profesionales y con más experiencia podrían tener opiniones diferentes. Por otro lado, el efecto de la habilidad del operador en la tasa de supervivencia de las restauraciones TRA es controversial ${ }^{10,12}$.

Una limitación importante de este estudio es que no se realizó el acompañamiento de las restauraciones realizadas por el método atraumático. Las familias que participaron de ese programa tenían el factor dificultador de no tener dirección fija en Goiânia o vivían en otras ciudades del Brasil Central. Se resalta que el impacto de los programas de salud oral cómo este, dirigidos para los niños hospitalizados, debe ser investigado en poblaciones mayores y a largo 
plazo, pues fue demostrado que el TRA realizado en servicios comunitarios gubernamentales puede elevar el número de dientes salvados por cuidados restauradores ${ }^{22}$. Sin embargo, nuestro principal objetivo con este estudio fue apuntar una nueva perspectiva para el problema de la mala condición oral en niños desfavorecidos socialmente.

Otro aspecto que deberá ser investigado futuramente es la relación entre el TRA, el control microbiano de la cavidad oral y la condición sistémica del paciente comprometido. Considerando la educación odontológica, un programa de salud oral para niños hospitalizados podría ayudar a llenar la laguna en relación a la preparación "de odontólogos generalistas para cuidar de bebés, pre-escolares y niños con necesidades especiales, así como de otros profesionales de salud y cuidadores para la promoción de la salud oral y prevención de enfermedades" ${ }^{23}$. Adicionalmente, la implantación de actividades de educación y prevención en salud oral, en ambiente hospitalario, puede permitir que los alumnos comprendan al paciente en su inte- gralidad y tengan una perspectiva más concreta de la inter relación entre la salud oral y la salud general ${ }^{18}$.

\section{Conclusiones}

Los odontólogos pueden contribuir para el desarrollo de programas de salud oral interdisciplinares en ambiente hospitalario y beneficiar comunidades carentes. Esos programas deben considerar la técnica del TRA para personas que no tienen acceso al tratamiento odontológico convencional, pues el presente estudio indicó que el TRA es posible en tales casos.

\section{Agradecimientos:}

Agradecemos a Ana Paula Pereira Castro, Daiene Granzotto Miranda, Fernanda Nogueira Silva, Kellen Cristina P. S. Parreira y Naiana P. Batista Oliveira por el apoyo a la colecta de datos.

Traducción: Fabian Calixto Fraiz; José Miguel Céspedes Amenábar

\section{Referencias}

1. Petersen PE. Global policy for improvement of oral health in the 21st century - implications to oral health research of World Health Assembly 2007, World Health Organization. Community Dent Oral Epidemiol 2009;37:1-8.

2. Junqueira SR, Pannuti CM, Rode SM. Oral health in Brazil - part I: public oral health policies. Braz Oral Res 2008;22:8-17.

3. Pedrazzi V, Dias KR, Rode SM. Oral health in Brazil - part II: dental specialty centers (CEOs). Braz Oral Res 2008;22:1823.

4. Maio MC, Lima NT. Fórum. O desafio SUS: 20 anos do Sistema Único de Saúde. Introdução. Cad Saude Publica 2009;25:1611-3.

5. Narvai PC, Frazão P, Roncalli AG, Antunes JLF. Cárie dentária no Brasil: declínio, polarização, iniqüidade e exclusão social. Rev Panam Salud Publica 2006;19:385-93.

6. Aujoulat I, Simonelli F, Deccache A. Health promotion needs of children and adolescents in hospitals: a review. Patient Educ Couns 2006;61:23-32.

7. Rezende GPSR, Costa LRRS, Cardoso RA. Pediatric dentistry during rooming-in care: evaluation of an innovative project for promoting oral health. J Appl Oral Sci 2004;12:149-53.

8. Medeiros Júnior A, Alves MS, Nunes JP, Costa IC. Outside clinical setting experience in a public hospital and oral health promotion. Rev Saude Publica 2005;39:305-10. 
9. Lo EC, Holmgren CJ, Hu D, van Palenstein Helderman W. Six-year follow up of atraumatic restorative treatment restorations placed in Chinese school children. Community Dent Oral Epidemiol 2007;35:387-92.

10. Frencken JE, van't Hof MA, Taifour D, Al-Zaher I. Effectiveness of ART and traditional amalgam approach in restoring single-surface cavities in posterior teeth of permanent dentitions in school children after 6.3 years. Community Dent Oral Epidemiol 2007;35:207-14.

11. Mickenautsch S, Yengopal V, Banerjee A. Atraumatic restorative treatment versus amalgam restoration longevity: a systematic review. Clin Oral Investig 2010;14:233-40.

12. Kemoli AM, van Amerongen WE. Influence of the cavity-size on the survival rate of proximal ART restorations in primary molars. Int J Paediatr Dent 2009;19:423-30.

13. Frencken JE. The ART approach using glass-ionomers in relation to global oral health care. Dent Mater 2010; 26:1-6.

14. WHO Collaborating Centre for Oral Health Care Planning and Future Scenarios. Basic Package for Oral Care. Nijmegen: University of Nijmegen; 2002.

15. Frencken JE, Phantumvanit P, Songpaisan Y, Pilot T. Manual for the atraumatic restorative treatment approach to control dental caries. Groningen: WHO Collaborating Centre for Oral Health Services Research; 1997.

16. Wright MC. Behavioural effects of hospitalization in children. J Paediatr Child Health 1995; 31:165-7.

17. Frankl SN, Shiere FR, Fogels HR. Should the parent remain with the child in the dental operatory? ASDC J Dent Child 1962; 29:150-63.

18. Curzon M. Supervised neglect - Again! Eur Arch Paediatr Dent 2010; 11:51-2.

19. Carvalho T, Ribeiro T, Bönecker M, Pinheiro E, Colares V. The atraumatic restorative treatment approach: an 'atraumatic' alternative. Med Oral Patol Oral Cir Bucal 2009; 14:e668-73.

20. Farag A, Frencken JE. Acceptance and discomfort from atraumatic restorative treatment in secondary school students in Egypt. Med Princ Pract 2009;18:26-30.

21. Wright GZ, Weinberger SJ, Marti R, Plotzke O. The effectiveness of infiltration anesthesia in the mandibular primary molar region. Pediatr Dent 1991;13:278-83.

22. Kikwilu EN, Frencken J, Mulder J. Impact of Atraumatic Restorative Treatment (ART) on the treatment profile in pilot government dental clinics in Tanzania. BMC Oral Health 2009;8:9-14.

23. Mouradian WE. The face of a child: children's oral health and dental education. J Dent Educ 2001;65:821-31.

Recibido: 27-09-2011

Envío evaluación: 29-09-2011

Aceptado: 18-10-2011

Correspondencia: 1sucasas@odonto.ufg.br 